\title{
Results from Experiments on Hybrid Plasma Immersion Ion Implantation/Nitriding Processing of Materials
}

\author{
M. Ueda ${ }^{1, a)}$, G. F. Gomes ${ }^{1)}$, K. G. Kostov ${ }^{1)}$, H. Reuther ${ }^{2)}$, C. M. Lepienski ${ }^{3)}$, \\ P. C. Soares Jr. ${ }^{3)}$, O. Takai ${ }^{4}$, and M. M. Silva ${ }^{5)}$ \\ 1) Associated Laboratory of Plasma, National Institute for Space Research, S.J. Campos, S.Paulo, Brazil \\ ${ }^{2)}$ Institute of Ion Beam Physics and Materials Research, Center Rossendorf, Dresden, Germany \\ 3) Department of Physics, Federal University of Paraná, Curitiba, Brazil \\ ${ }^{4)}$ Department of Materials Processing Engineering, Nagoya University, Chikusa, Nagoya, Japan \\ ${ }^{5)}$ Department of Mechanical Engineering, Technological Institute of Aeronautics, S.J.Campos, S.Paulo, Brazil
}

Received on 21 January, 2004; revised version received on 22 April, 2004

\begin{abstract}
To improve the performance of critical part components, new methods for surface strengthening are being developed with success, like plasma immersion ion implantation (PIII) and hybrid surface treatments mixing PIII and ion nitriding processes. A combination of high pressure $\left(4 \times 10^{1} \mathrm{~Pa}\right)$, moderate temperature (up to $450^{\circ} \mathrm{C}$ ) glow discharge nitriding with low pressure $\left(8 \times 10^{-2} \mathrm{~Pa}\right)$ and low DC bias voltage ion nitriding (or DC PIII) was implemented. Depending on the particular conditions of the treatment and the depth probed, mixed phases of $\gamma_{N}$ and $\varepsilon$ were measured in the treated SS304 steel sample. This near surface modification resulted in an improved hardness (up to a factor of $2.7 \times$ ) of the sample which could also enhance its wear properties. Surface modification of Ti6Al4V alloy and SS304 steel by a combination of PIII and subsequent ion nitriding was investigated as well. Nitrogen ions were implanted into the specimens at $15 \mathrm{keV}$ and then ion nitrided at low pressure $\left(7 \times 10^{-2} \mathrm{~Pa}\right)$ with a bias of $-800 \mathrm{~V}$. Compared to the untreated samples, the hardness of Ti6Al4V alloy and the steels could be improved significantly. AES results indicated high retained doses in both samples, confirming the high efficiency of this hybrid process.
\end{abstract}

\section{Introduction}

Ion nitriding is a well known surface modification technique used extensively in the modern industries to increase the hardness and wear resistance of selected metal components. Conventional nitriding of workpieces is carried out in an atmosphere of partially dissociated ammonia or in a cyanide-cyanate salt bath, at temperatures of $650-850^{\circ} \mathrm{C}$. Both methods present not only high potential for environmental hazards but also are prone to causing severe industrial accidents involving operators during the treatment. Furthermore, difficulties in controlling the "white layer" are another disadvantage of these methods of nitriding.

Ion nitriding using plasma, on the other hand, is a much more advanced method of uptaking nitrogen into the metal component surface with benefits regarding environmental aspects, control of "white layer", reduced distortion of the workpiece and energy consumption, etc [1]. In the conventional ion nitriding of steels, temperatures over $500^{\circ} \mathrm{C}$ are required to reach a satisfactory nitriding result $[2,3]$. The components under treatment are used as cathodes of the discharges that can be run at the DC (typically 700 to $1500 \mathrm{~V}$ ) or pulsed mode (with frequencies of up to tens of kilohertz). The nitrogen ions and atoms present in the plasma are mostly absorbed on the surface of the components, and by means of complex physical and chemical processes therein being thermally diffused into deep layers, ranging from a few to tens of $\mu \mathrm{m}$, in treatments as long as tens of hours [3].

New techniques for surface strengthening are being further developed with success, like plasma immersion ion implantation, PIII [4,5], and also hybrid surface treatments combining PIII with other traditional techniques of deposition, electroplating, etc. [6]. A recent work in this direction was presented elsewhere, exploring the hybrid process mixing PIII and ion nitriding [7]. In particular, a combination of high pressure, high temperature glow discharge nitriding with low pressure ion nitriding was successfully implemented recently $[7,8]$. The low pressure phase of this treatment was carried out in the same way as the so called DC PIII, as will be shown clearly later in this paper.

Another possibility explored in this work deals with a related hybrid treatment using PIII with subsequent low pressure nitriding [9]. This surface pre-treatment with high energy ion bombardment and sputtering of the target surface during the PIII process may create appropriate conditions for an efficient subsequent nitriding [10,11]. This can be important for some applications, for instance, in the case of Ti6Al4V, which is widely used as biomaterial due to its high strength-to-weight ratio, excellent corrosion resistance, and exceptional biocompatibility. However, the tribological properties of this titanium alloy are notoriously poor. Nitrogen ion implantation improves the wear resistance of 
Ti6Al4V, however, the thickness of the improved layer formed by traditional ion implantation may not be adequate for many medical or industrial applications [12]. Substantially thicker modified layer exhibiting more superior wear resistance could be obtained in Ti alloy if much higher temperatures (as high as $800^{\circ} \mathrm{C}$ ) can be achieved during PIII or by using other auxiliary heating methods. The surface pretreatment with ion implantation is also a different possibility for improved thickness of treated layer [11].

In this paper, we discuss some aspects of hybrid treatments on metallic materials based on PIII combined with low pressure ion nitriding and DC PIII mixed with high pressure ion nitriding, focusing mainly on the results obtained on materials surface after the applications of these hybrid processes.

\section{Experimental}

The schematic drawing of the experimental devices used for the hybrid processes are shown in Fig. 1. In this figure we show both: the case of hybrid process using alternated high pressure nitriding and low pressure DC PIII, in which we alternated the use of DC bias for the nitriding and biasing in Feed 2, and the case of hybrid process using PIII followed by nitriding, in which we alternated the HV pulser and DC bias in the Feed 2. Most components used for both processes are the same but in the first a high pressure discharge is turned on to obtain a cathodic plasma on the sample support by feeding DC negative voltage ( -600 to $-800 \mathrm{~V}$ ) through the power supply indicated by main glow (Feed 2), in Fig. 1. After reaching temperatures of $400-500^{\circ} \mathrm{C}$, a low pressure diffuse plasma is generated in substitution to the cathodic plasma by driving a glow discharge through the feed 1 while maintaining a DC negative bias of $700 \mathrm{~V}$ in the sample support.

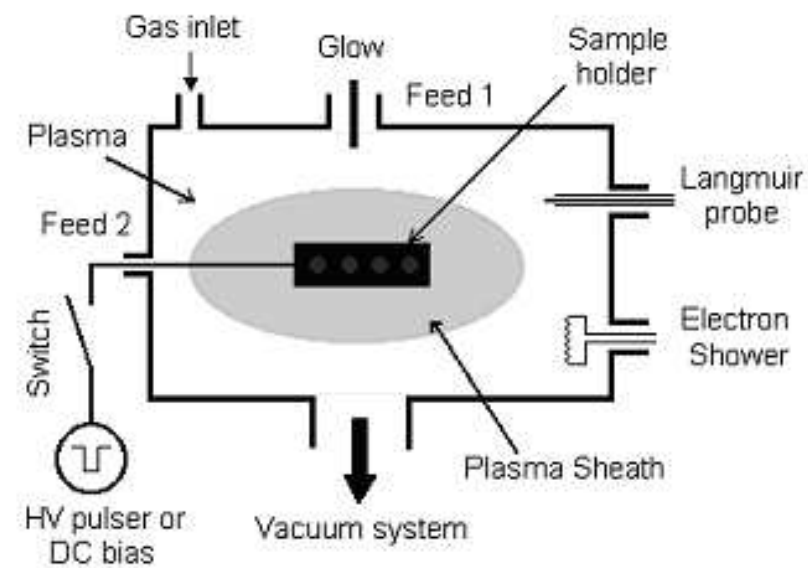

Figure 1. Schematic drawing of the experimental set-up for hybrid alternated high and low pressure nitriding and for PIII followed by low pressure nitriding processing of materials.

On the other hand, for the second hybrid process discussed here, the feed 2 is used to drive the PIII treatment process while the glow discharge is run through the feed 1. For the subsequent nitriding, the DC bias is fed through feed
2 while the plasma is produced using the feed 1. Other components shown in the figures are common to both processes and have been discusses in previous papers [5].

The temperatures of the samples during the treatments (very critical for the first hybrid process) are carefully monitored using a RAYTEK infrared pyrometer, sensitive in the $200-2000^{\circ} \mathrm{C}$ range.

For the measurement of x-ray diffraction (XRD) of nitrided SS304 and Ti6Al4V, Philips 3410 and PW3710 diffractometers in the standard $2 \theta$ mode were used.

For the AES (Auger Electron Spectroscopy) measurements used for determination of the elemental concentration in the samples, a spectrometer from FISONS Instruments Surface Science, model MICROLAB 310-F was used.

Tribological evaluations of the samples surface were conducted with a Tribo Scope nanomechanical indentation tester from Hysitron Inc.

Glow discharge optical spectroscopy (GDOS) depth profiling analysis was performed in a JY-5000RF (Jobin-Yvon) device.

Surface morphology of the Ti alloy samples was analyzed by atomic force microsopy (AFM) operating the Shimadzu SPM - $9500 J 3$ nanoindenter in the dynamic mode.

\section{Results and discussions}

\subsection{Part 1}

Results on SS304 samples exposed to the first hybrid process, i.e. alternated high pressure nitriding and low pressure DC PIII will be discussed first. The discharge conditions were: for high pressure nitriding, the discharge was run at pressures of $30-40 \mathrm{~Pa},-600$ to $-800 \mathrm{~V}$ and currents of 250 $\mathrm{mA}$, while for low pressure nitriding, the discharge was run at $8 \times 10^{-2} \mathrm{~Pa}, 900 \mathrm{~V}, 80 \mathrm{~mA}$, respectively. For the first cycle of the treatment, the plasma is produced using the sample support as the cathode. On the other hand, for the second cycle, the plasma is produced away from the sample support and it fills the chamber by self-diffusion while a bias voltage of $-700 \mathrm{~V}$ is applied to the support to attract the ions. The working gas in this case was a mixture of nitrogen and hydrogen in the 1:1 ratio. These two cycles of 15 min each were repeated for 5 times, completing $150 \mathrm{~min}$ in total.

In Fig. 2(b) we show an XRD diffractogram obtained for SS304 treated by this hybrid process while in Fig. 2(a) a reference XRD for the standard SS304 sample is shown. A very prominent $\gamma_{N}$ broad peak can be seen to the left of normal $\gamma(111)$ peak of Fe in Fig.2 (b), indicating a thick layer of the $\gamma_{N}$ phase. This is the so called expanded austenite phase (or high concentration of nitrogen in solid solution) which presents excellent tribological properties as high hardness and resistance to wear. Indeed, the reached hardness in this case was $542 \mathrm{HV}$ (an enhancement factor of $2.7 \times$, compared to an untreated sample) which was confirmed by nanoindentation hardness profile, as shown in Fig. 3. The hardness profile obtained shows that near $150 \mathrm{~nm}$ the increase of hardness is about $2.7 \times$ while even for $500 \mathrm{~nm}$ this factor is over $2.0 \times$. In fact, at the depths lower than $100 \mathrm{~nm}$, the measurements indicate a decrease of the enhancement factor which 
could be due to a highly damaged surface (as was seen by glancing XRD results shown in a previous paper [13]).

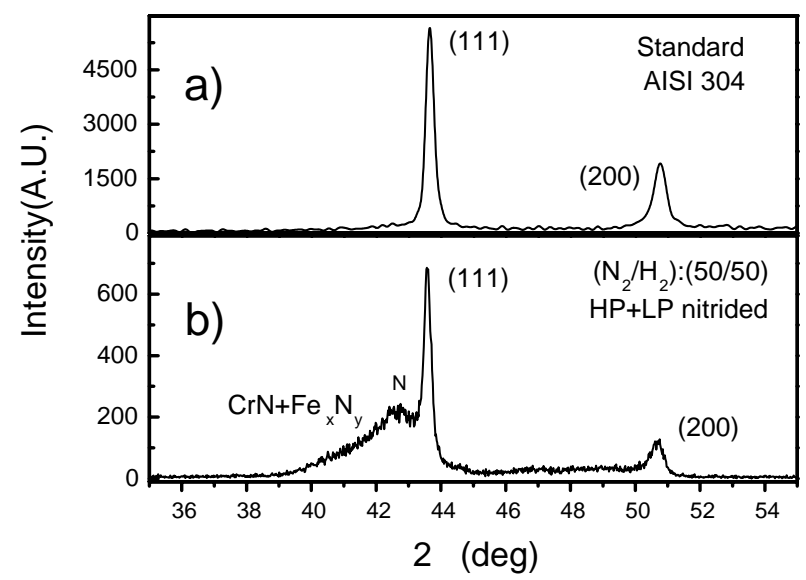

Figure 2. Normal incidence XRD results for: (a) standard SS304; (b) $\left(\mathrm{N}_{2} / \mathrm{H}_{2}\right)$ : (50/50) gas mixture nitrided $\mathrm{SS} 304$.

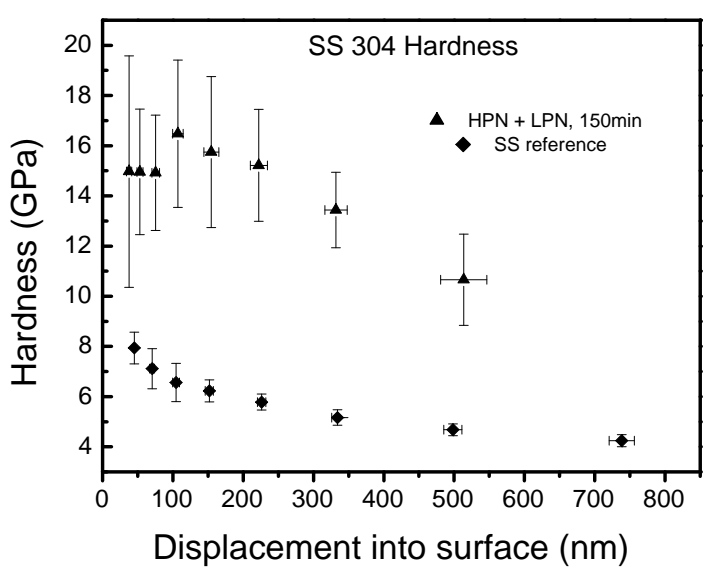

Figure 3. Hardness profiles of standard SS304 sample and one treated by hybrid high and low pressure nitriding processing.

The AES profiling obtained for this treatment can be seen in Fig. 4. Here we can see that the hybrid treatment enabled us to inject a huge amount of nitrogen into the $\mathrm{Fe}$ structure. More than $30 \%$ of the elements detected at 25 $\mathrm{nm}$ depth are nitrogen and even at $650 \mathrm{~nm}$ this percentage is over $20 \%$. GDOS measurements (not shown here) confirmed both glancing XRD[13] as well as AES results and indicated a nitrogen penetration of more than $2 \mu \mathrm{m}$. Notice also that the amount of impurities introduced by this process is very low, concentrating them at the very surface of the samples $(<10 \mathrm{~nm})$.

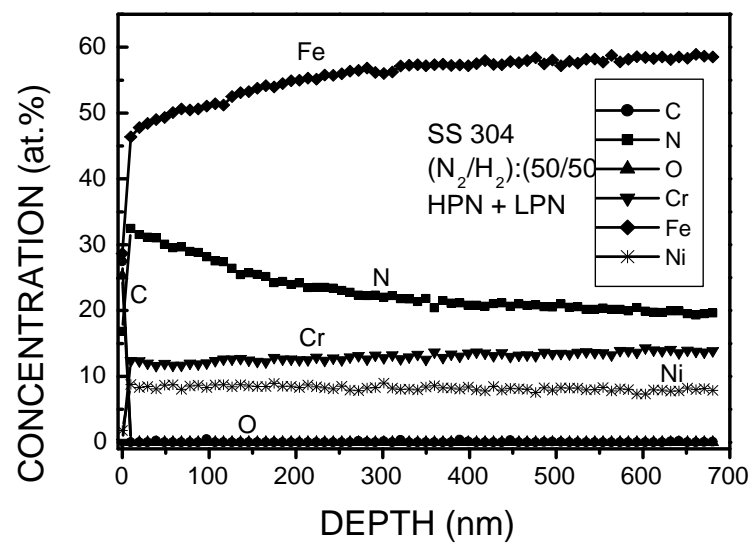

Figure 4. AES profiles of SS304 treated under a plasma condition of $\left(\mathrm{N}_{2} / \mathrm{H}_{2}\right)$ : $(50 / 50)$ gas mixture, nitrided with alternating high pressure and low pressure cycles.

\subsection{Part 2}

In this second part, we will discuss the results obtained by a hybrid process involving higher energy PIII (10 to $15 \mathrm{kV}$ ) combined with a low pressure nitriding, applied to SS304 and Ti6Al4V alloy.

Compact oxide layer on the surface of metal alloys is a strong barrier for the nitrogen diffusion into the bulk material. This prevents the effective use of ion nitriding in many types of metal alloys. PIII at moderated energy (10 - $30 \mathrm{keV}$ ) allows ion penetration depths deeper than these surface oxide layers. The damages caused by ion implantation and the surface sputtering in these outermost layers may create favorable boundary conditions for an efficient subsequent diffusive treatment such as ion nitriding.

We will start our discussion on results of this hybrid treatment applied to SS304. In a previous paper we have discussed the formation of a double layer of expanded austenite $\gamma_{N}$ 's $\left(\gamma_{N 1}\right.$ and $\left.\gamma_{N 2}\right)$ when this process was used in SS304 [9]. This result can be confirmed by the AES results shown in Fig. 5(a) and (b), where we compare the AES profiles for the case when only PIII was applied and for the case with hybrid process in which PIII and nitriding was used. For the first treatment, the implantation parameters were: pulse width of $20 \mu \mathrm{s}$, pulse repetition rate of $400 \mathrm{~Hz}$, and implantation voltage of $15 \mathrm{kV}$. The treatment total time was $150 \mathrm{~min}$ and the temperatures of the samples reached $450^{\circ} \mathrm{C}$. For the second treatment we used the same implantation parameters, however, the total implantation time was only 75 min which was followed by $75 \mathrm{~min}$ of ion nitriding at low pressure with a bias of $-700 \mathrm{~V} \mathrm{DC}$. The temperature at the beginning of nitriding phase was $420^{\circ} \mathrm{C}$ which decreased rapidly (it reached $250^{\circ} \mathrm{C}$ in $10 \mathrm{~min}$ ). 


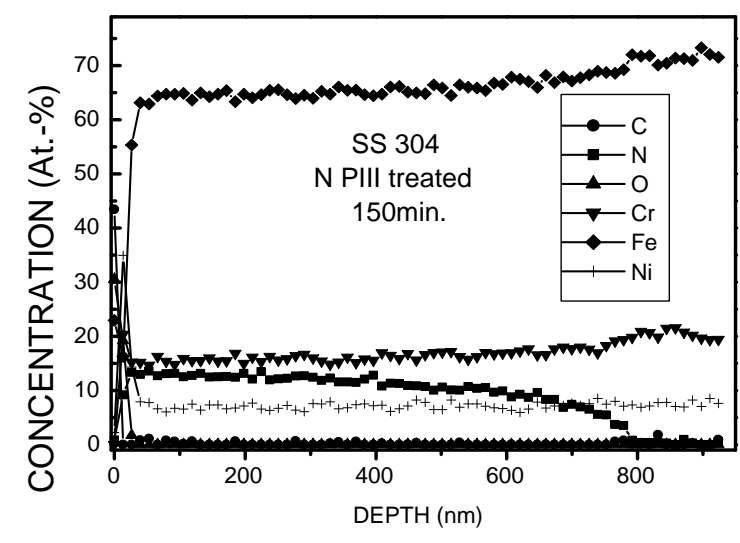

Figure 5(a). AES profiles of SS304 sample treated under a nitrogen plasma PIII for $150 \mathrm{~min}$.

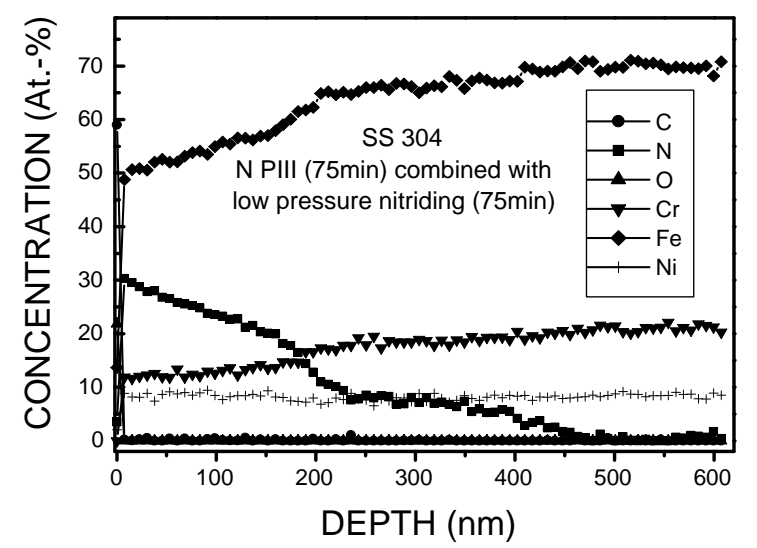

Figure 5(b). AES profiles of SS304 sample treated under a nitrogen plasma PIII ( $75 \mathrm{~min})$ combined with low pressure ion nitriding (75 min).

In Fig. 5(a) we can see that a deep nitrogen penetration (800nm) was achieved by the PIII alone, applied for 150 min. This was due to a good thermal diffusion of the implanted nitrogen in SS304 at temperatures of $450^{\circ} \mathrm{C}$. The percentage of nitrogen introduced into the steel is typically $15 \%$. Compared to that behavior, the result of Fig. 5(b) shows that a treatment with 75 min PIII followed by 75 min nitriding causes an increase of nitrogen percentage in the shallower regions. It reaches percentages over $20 \%$ between $10 \mathrm{~nm}$ to $150 \mathrm{~nm}$. This enriched nitrogen layer sits on top of the layer with about $10 \%$ nitrogen concentration that extends over to $450 \mathrm{~nm}$, which is the result of PIII treatment alone. This double layer in concentration reflects into double layer in $\gamma_{N}$ phases. This kind of structures might be useful for some types of application where ability to support very high loads is required. Indeed, it was shown in a previous paper that the most adequate hardness profile (higher hardness to deeper depths and much higher hardness at the very surface) is obtained by using the hybrid process [9].
Next we will discuss the results obtained with this hybrid process (PIII followed by nitriding) applied to the $\mathrm{Ti}$ alloy. The effect of $\mathrm{TiO}_{2}$ formed on the surface of the sample of this alloy may pose more difficulties for nitriding than in the case of the nitriding of steels. The thermal diffusion of nitrogen in this alloy requires also much higher temperatures, of the order of $800^{\circ} \mathrm{C}$. Hence an alternative nitrogen diffusion process would be welcome to enhance its surface properties when such high temperatures can not be obtained so easily. In the previous experiments, we had obtained enhancement in hardness (up to $70 \%$ ) and reduction of friction coefficient (down to 1/3) when we applied solely nitrogen PIII for up to $2 \mathrm{~h}$, with pulsing voltages of $11 \mathrm{kV}, 50 \mu$ s duration and 400 $\mathrm{Hz}$ repetition frequency [14]. In the present experiment, PIII and nitriding were combined in the same way as in the case of SS304 treatment.

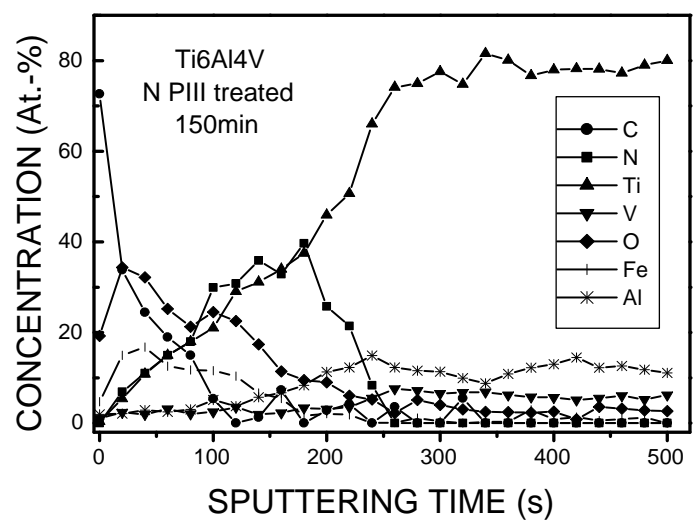

Figure 6(a). AES profiles of Ti6Al4V sample treated under a nitrogen plasma PIII for $150 \mathrm{~min}$.

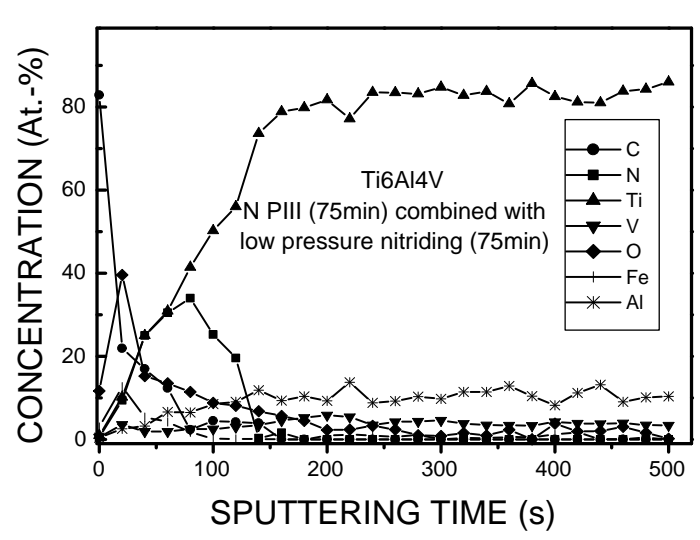

Figure 6(b). AES profiles of Ti6Al4V sample treated under a nitrogen plasma PIII ( $75 \mathrm{~min}$ ) combined with low pressure ion nitriding (75 min).

In Fig. 6(a) we show the AES profiling for the case with only PIII (150 min). Nitrogen implantation of up to $40 \%$ atomic concentration is achieved with a penetration to about $65 \mathrm{~nm}$. When PIII of $75 \mathrm{~min}$ is combined with nitriding of 
75 min, an AES result as shown in Fig. 6(b) is obtained. Nitrogen uptake is not increased during the nitriding phase. Instead we notice some sputtering effect in that phase. Indeed, when we increased the nitriding time to $4 \mathrm{~h}$, the initially nitrogen implanted layer was all sputtered away. AFM photos (not shown here) revealed that the Ti6Al4V sample with result of Fig.6 (b) had a much smoother surface than sample of Fig.6(a).

The nanoindentation measurements for these two processes showed hardness profile as shown in Fig. 7. Here we also included the data for PIII and $4 \mathrm{~h}$ nitriding. We can see a hardness improvement of about $50 \%$ for all the treatments under discussion. This independence of hardness values with nitrogen implanted profile is a well known effect in the field of ion implantation, the cause of this improvement being attributed to some damages and dislocations occurring in front of the implanted layer.

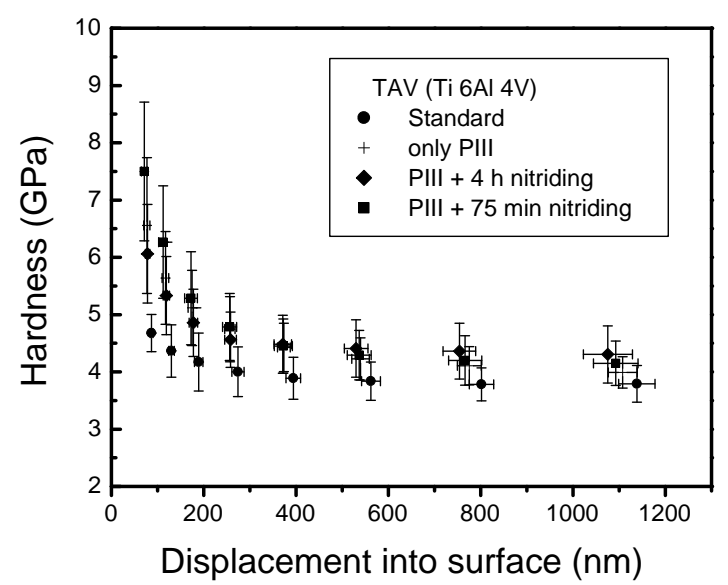

Figure 7. Hardness profiles of standard Ti6A14V sample, one treated by PIII alone, one treated by PIII combined with nitriding ( 75 $\mathrm{min})$ and one treated by PIII and nitriding (4 h).

We expect that the smoother surface of Ti alloy obtained by the hybrid PIII combined with nitriding process leads to a much lower friction coefficient due to its smoother surface. Unfortunately the hardness increase seems to be saturated at 75 min nitriding condition. A PIII treatment at higher temperatures (of order of $800^{\circ} \mathrm{C}$ ) should increase the treated layer thickness and when combined with nitriding may produce a final surface with lower friction coefficient.

\section{Conclusion}

Two hybrid material processing methods were developed. In one of them, ion nitriding of SS 304 at high pressure was combined with a low pressure nitrogen DC PIII and applied to take advantages of high temperature (near $450^{\circ} \mathrm{C}$ ) achieved in the high pressure phase and of effective ion acceleration and deeper implantation by the bombardment of ions with energies near $0.7 \mathrm{keV}$ at the low pressure phase. This treatment led to a very efficient ion implantation and diffusion of nitrogen (about $2 \mu$ m penetration) in SS 304 and as a result, formation of a thick layer with $\gamma_{N}$ phase with improved hardness (factor of $2.7 \times$ over the reference sample) and low surface contamination.

In the second experiment, hybrid treatment combining nitrogen PIII and subsequent nitriding was attempted in two different materials (SS 304 and Ti6Al4V). Although a deep treatment was not achieved by this process, as suggested by previous paper on results of ion beam combined with nitriding processing[11], we obtained a few new results in surface modification by plasma and PIII ion implantation. For SS 304, a double layer with different $\gamma_{N}$ 's $\left(\gamma_{N 1}\right.$ and $\left.\gamma_{N 2}\right)$ were obtained, according to XRD diffraction and AES profiling results. A double layer with a very high hardness profile at the very surface obtained by nitriding and a deep layer with moderate hardness obtained by PIII, could lead to a new material surface with high strength capable of supporting high load.

In the case of Ti6Al4V sample exposed to this method of hybrid surface modification, it was found that PIII alone produces sample with a shallow implanted layer (about $65 \mathrm{~nm}$ ) with high hardness, the surface of which is sputtered quite strongly during subsequent ion nitriding. Little uptake of nitrogen occurs during this nitriding phase but a smoothening of the surface results which can reduce the friction coefficient. Hence a new attempt will be made combining a high temperature PIII with ion nitriding finishing which could result in a thick, high hardness and low friction Ti6Al4V surface, much desired in the industrial applications of this material.

\section{Acknowledgements}

We thank to FAPESP and CNPq for financial support. Two of us, GFG and KGK thank to FAPESP for research grants.

\section{References}

[1] In Plasma Science and Technology by Herman V. Boenig, Cornell University Press, Ithaca and London, 1982.

[2] B. Larish, U. Brusky, and H. J. Spies. Surf. Coat. Techn. 116119, 205 (1999).

[3] In Surface Modification Engineering, Vol.II, Ed. Ram Kossovsky, CRC Press Inc., Florida, 1989.

[4] In Handbook of Plasma Immersion Ion Implantation and Deposition, Ed. André Anders, J. Wiley\&Sons Inc., 1st ed., Toronto, 2000.

[5] M. Ueda, L. A. Berni, G. F. Gomes, A. F. Beloto, E. Abramof, and H. Reuther. J. Appl. Phys. 86, 4821 (1999).

[6] W. Ensinger, K. Voltz, and B. Enders, Surf. Coat. Techn. 120121, 343 (1999).

[7] M. Ueda, G. F. Gomes, E. Abramof, and H. Reuther, Nucl. Instrum. Meth. B206, 749 (2003).

[8] G. F. Gomes, M. Ueda, and H. Reuther, J. Appl. Phys. 94, 5379 (2003). 
[9] K. Kostov, M. Ueda, M. Lepienski, P. C. Soares Jr, G. F. Gomes, M. M. Silva, and H. Reuther, Surf. Coat. Technol. 186/1-2, 204 (2004).

[10] I. Kanno, K. Nomoto, S. Nishijima, T. Nishiura, and T. Okada, Nucl. Instrum. Meth. B 59-60, 920 (1991).

[11] N. Nunogaki, H. Suezawa, Y. Kuratomi, and Y. Miyazaki, Vacuum 39, 281 (1989).
[12] S. Y. Wang, P. K. Chu, B. Y. Tang, X. C. Zeng, Y. B. Chen, and X. F. Wang, Surf. Coat. Technol. 93, 309 (1997).

[13] M. Ueda, G. F. Gomes, E. Abramof, and H. Reuther, Surf. Coat. Technol. 186/1-2, 291 (2004).

[14] M. Ueda, M. M. Silva, C. Otani, H. Reuther, M. Yatsuzuka, C.M. Lepienski, and L. A. Berni, Surf. Coat. Technol. 169170, 408 (2003). 